\title{
Elementary Interactions in Quasi-linear Hyperbolic Systems
}

\author{
Cleve Moler \& Joel Smoller \\ Communicated by L. CESARI
}

\begin{abstract}
1. Introduction
We consider the existence of solutions of the Cauchy problem for a $2 \times 2$ quasi-linear hyperbolic system

$$
U_{t}+F(U)_{x}=0, \quad U(0, x)=U_{0}(x),
$$

where $t \geqq 0,-\infty<x<\infty, U(t, x)=(u(t, x), v(t, x))$, and the function $F(U)=$ $(f(u, v), g(u, v))$ is smooth. GLIMM [2] has proved existence of a weak solution provided that the variation of the initial data $U_{0}(x)$ is small. GLIMM \& LAX [3] then improved this result by requiring that the oscillation of $U_{0}(x)$ be small. SMOLLER [7] has proved the existence of a weak solution provided that $U_{0}(x)$ is constant except for a single arbitrary jump (the Riemann problem). Finally, NisHida [6] has proved existence for general data $U_{0}$ but for the special case $f=1 / v, g=u$.
\end{abstract}

Here we will prove existence under the assumption that $U_{0}(x)$ is constant except for two jumps; that is, $U_{0}(x)$ is of the form

$$
U_{0}(x)= \begin{cases}U_{l}, & -\infty<x<x_{1}, \\ U_{m}, & x_{1}<x<x_{2}, \\ U_{r}, & x_{2}<x<\infty\end{cases}
$$

Furthermore, we assume that these constant states $U_{l}, U_{m}$ and $U_{r}$ are separated by two waves, which however can be of arbitrary strength. Thus, the Riemann problem with initial states $U_{l}$ and $U_{m}$ would generally produce two shocks or rarefaction waves separated from each other by a third constant state $U_{3}$. Since we are only interested in the problem of elementary interactions, we will assume that $U_{l}$ and $U_{m}$ are related in such a way that only one such wave is produced and hence that $U_{3}$ would actually be equal to $U_{l}$ or $U_{m}$. This means that the point $U_{m}$ must lie on one of four specified curves in the $u-v$ plane through $U_{l}$ (the shock or rarefaction wave curves, cf. [7]), but that the distance from $U_{m}$ to $U_{l}$ may be arbitrarily large. We assume that $U_{r}$ and $U_{m}$ are similarly related. Our object is to study the interaction that occurs when one of the waves overtakes the other, i.e., the initial waves move in such a way that they intersect destroying 
$U_{m}$. Our assumptions are the simplest which allows this to occur. We remark that these interactions are of considerable interest in gas dynamics; cf. [1] and the references given there.

The method we use is the Glimm difference scheme [2]. We programmed Glimm's scheme for a computer and calculated approximate solutions for various functions $F$ and initial data $U_{0}$. Observation of certain patterns in the behaviour of the computed solutions led to the proof of the existence theorem, which we give here.

If the system (1) is hyperbolic and the initial data (2) satisfies our conditions, then each of the initial discontinuities produces one of four waves ${ }^{1}$ : a backward rarefaction wave, a backward shock wave, a forward rarefaction wave or a forward shock wave. This gives sixteen qualitatively different situations to consider. Of these, six are trivial because the waves never meet and there is no actual interaction. Of the remaining cases, three involve the interaction of only shocks and are effectively Riemann problems at the time of intersection. These were studied in [7] and [8]. Two cases involve a shock interacting with a rarefaction wave of the "opposite direction" and are considered in [8]. One involves the intersection of two rarefaction waves and is studied in [4] or [9]. This leaves the four cases which we consider here: a forward rarefaction wave overtaking a forward shock wave, a forward shock wave overtaking a forward rarefaction wave, and the two symmetrically equivalent interactions involving backward waves. In these situations we shall show that although the actual solution may be rather complicated (involving the formation and breaking of compression waves) the solution is contained in a bounded region determined by the initial conditions, and the variation of the solution on any line $t=$ const. $>0$ is bounded by a constant depending only on the initial conditions.

We assume that $f_{v} g_{u}>0$, and for definiteness we take $f_{v}<0$ and $g_{u}<0$. We also assume that (1) satisfies the following condition: if $l_{i}$ and $r_{i}, i=1,2$, are the left and right eigenvectors of $d F$ normalized so that $d \lambda_{i}\left(r_{i}\right)>0$ and $l_{i} r_{i}>0$, then $l_{i} d^{2} F\left(r_{j}, r_{j}\right)>0, i, j=1,2$. (This is equivalent to assuming that the system (1) is genuinely nonlinear and satisfies the shock interaction conditions [8].) These conditions were studied in [7] and [8] and imply certain convexity and monotonicity properties of the shock and rarefaction wave curves.

Finally we assume the shock stability conditions ([7]) which state that $\lambda_{1}(U(t, x-0))<\sigma$ holds across 2-shocks and $\lambda_{2}(U(t, x+0))>\sigma$ holds across 1 -shocks where $\sigma$ is the shock speed.

We can now state our theorem.

Theorem. Assume that the system (1) satisfies the above conditions and that the initial data (2) is so chosen that each initial discontinuity produces only a single wave. Then the problem (1)-(2) has a solution for all $t>0$. This solution is bounded and has uniformly bounded total variation on each line $t=$ const. $>0$.

\footnotetext{
${ }^{1}$ Under the assumption of hyperbolicity, the Jacobian $d F$ has real and distinct eigenvalues $\lambda_{1}(u, v)<\lambda_{2}(u, v)$. We shall say that a wave is "backward" if it is associated with $\lambda_{1}$ even though $\lambda_{1}$ is not necessarily negative. Similarly "forward" waves are those associated with $\lambda_{2}$.
} 
We remark that our solutions are, of course, weak solutions in the usual sense of the theory of distributions.

\section{Numerical Experiments}

Although it was probably not intended for actual computation, Glimm's difference scheme can be implemented on a computer, and we have found it very useful as an experimental tool for the study of the complex interactions which occur in this problem.

The Glimm scheme involves mesh parameters $h=\Delta t$ and $k=\Delta x$. In the proof of the theorem we will require that

$$
k / h>\sup \left\{\left|\hat{\lambda}_{j}(U)\right|, U \in B, j=1,2\right\}
$$

where $B$ is a compact set known to contain the solution. This condition is analogous to the well-known numerical stability condition $k / h>c$ necessary for the simple difference approximation to the linear wave equation $w_{t t}-c^{2} w_{x x}=0$ (or if $u=w_{t}, v=c w_{x}$, the system $v_{t}-c u_{x}=0, u_{t}-c v_{x}=0$ ). But in actual computation with the Glimm scheme, (3) apparently plays a somewhat different role. Even when it is not satisfied, experiments indicate that round-off errors and rapid changes in the solution do not exhibit exponential growth.

The scheme defines a function $V(x, t)$ which is a solution to the system on each horizontal strip $n h<t \leqq(n+1) h$. This solution is represented at mesh points ( $m k, n h)$ by values $U_{m, n}$ which approximate $U(m k, n h)$. Only $m$ and $n$ for which $m+n$ is even are used. For $n=0, U_{m, 0}=U_{0}(m k)$. For $n+1>0$, the value of $U_{m, n+1}$ is calculated using $U_{m-1, n}, U_{m+1, n}$ and a random variable $a=a_{m, n}$ drawn from a uniform distribution $-1 \leqq a \leqq 1$. Specifically, $U_{m, n+1}=V((m+a) k,(n+1) h)$ where $V(x, t)$ is defined, for $(m-1) k \leqq x \leqq(m+1) k, n h \leqq t \leqq(n+1) h$, to be the solution of the Riemann problem (1) with initial data $U(x, n h)=U_{m-1, n}$, if $x<m k$ and $U(x, n h)=U_{m+1, n}$, if $x>m k$. The solution of the Riemann problem generally requires computing the intersection of two curves defined by a pair of ordinary differential equations. Since this is done for each $m$ and $n$, the Glimm scheme obviously requires much more computation than standard difference methods.

We have only experimented with the special systems $u_{t}-v_{x}=0, v_{t}+f(u)_{x}=0$, where $f^{\prime}<0$ and $f^{\prime \prime}>0$. Here the Riemann problems can be solved relatively easily. Since $-\lambda_{1}(u)=\lambda_{2}(u)=\left[-f^{\prime}(u)\right]^{\frac{1}{2}}$, we define

and

$$
\lambda(u)=\left[-f^{\prime}(u)\right]^{\frac{1}{2}}, \quad \rho\left(u_{1}, u_{2}\right)=\left|\int_{u_{1}}^{u_{2}} \lambda(u) d u\right|,
$$

$$
\sigma\left(u_{1}, u_{2}\right)=\left[\left(u_{1}-u_{2}\right)\left(f\left(u_{2}\right)-f\left(u_{1}\right)\right)\right]^{\frac{1}{2}} \text {. }
$$

The functions $\rho$ and $\sigma$ characterize the rarefaction and shock wave curves respectively. 


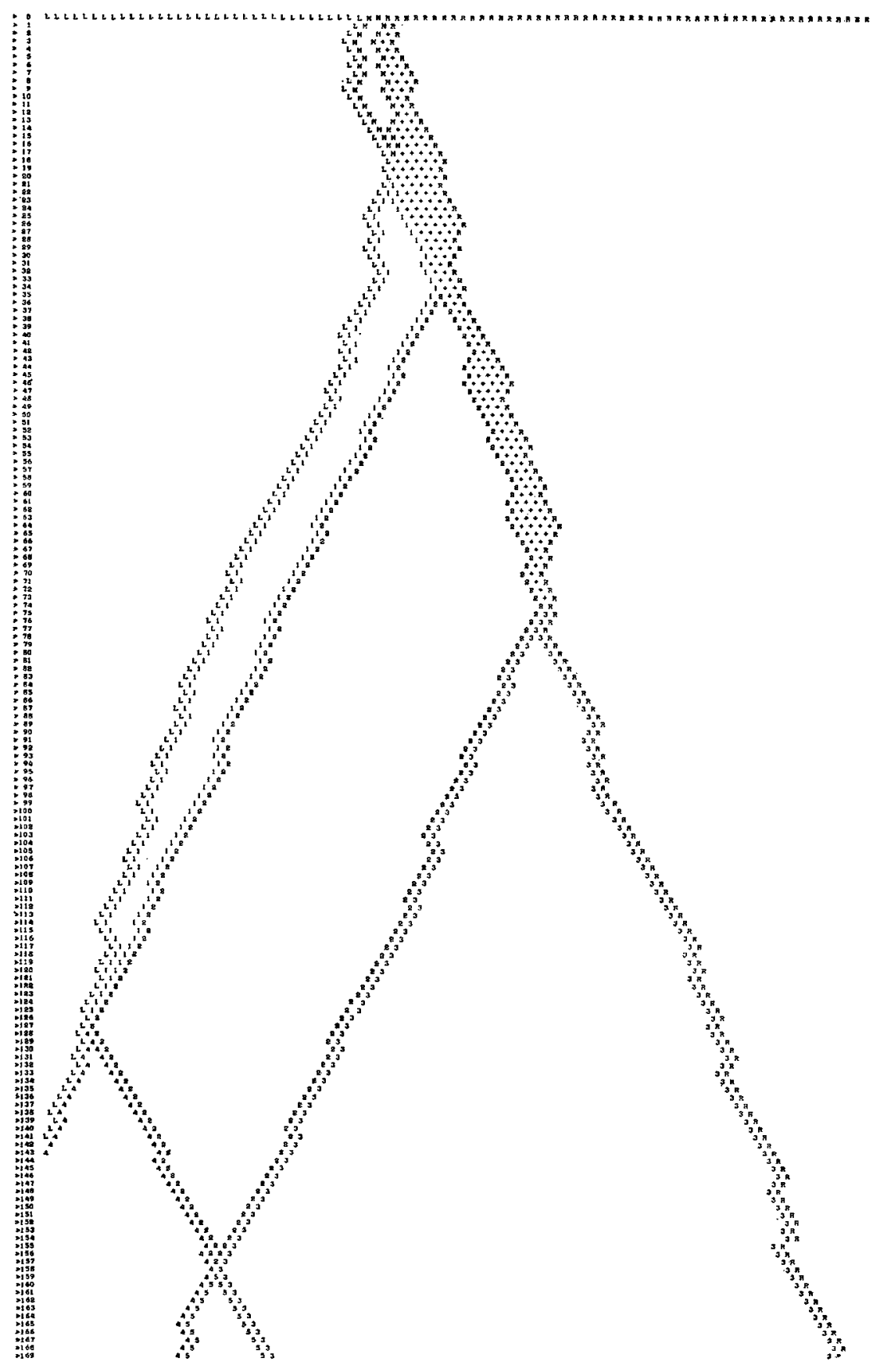

Fig. 1 
To compute $U_{m, n+1}$, let $\left(u_{l}, v_{l}\right)=U_{m-1, n},\left(u_{r}, v_{r}\right)=U_{m+1, n}, \rho=\rho\left(u_{l}, u_{r}\right)$, $\sigma=\sigma\left(u_{l}, u_{r}\right)$ and consider four cases:

I) $-\sigma \leqq v_{r}-v_{l} \leqq \rho, u_{l} \leqq u_{r}$ (back rarefaction, front shock)

II) $v_{r}-v_{l} \leqq-\sigma \quad$ (back shock, front shock)

III) $-\sigma \leqq v_{r}-v_{l} \leqq \rho, u_{r} \leqq u_{l}$ (back shock, front rarefaction)

IV) $\rho \leqq v_{r}-v_{l} \quad$ (back rarefaction, front rarefaction).

If equality occurs, either choice may be made and one of the resulting waves has zero strength. (Our restriction on $U_{0}$ implies that equality occurs initially.) For illustration, consider case I. Compute the intermediate state $(u, v)=U$ by determining the intersection of the two curves $v=v_{l}+\rho\left(u_{l}, u\right)$ and $v=v_{r}+\sigma\left(u, u_{r}\right)$. Generate a pseudorandom number $\xi$ in the interval $[-k / h, k / h]$. Then define $U_{m, n+1}$ to be $U_{l}$ if $\xi \leqq-\lambda\left(U_{l}\right), U_{r}$ if $\sigma_{r}=\sigma\left(u_{r}, u\right) /\left(u_{r}-u\right) \leqq \xi$, or $U$ if $-\lambda(u) \leqq \xi \leqq \sigma_{r}$. Otherwise, if $-\lambda\left(u_{l}\right) \leqq \xi \leqq-\lambda(u)$, let $U_{m, n+1}=\left(u_{\xi}, v_{\xi}\right)$, where $u_{\xi}=\lambda^{-1}(\xi)$ and $v_{\xi}=v_{l}+\rho\left(u_{l}, u_{\xi}\right)$ represent an intermediate state in a rarefaction wave joining $U_{l}$ and $U$. This completes case $\mathrm{I}$; the others are similar and will be omitted.

Fig. 1 illustrates the computer output for a typical problem. The symbols stand for different states at points in the $x-t$ plane. The letters $L, M$ and $R$ are the initial states, the digits and other letters are new states produced by interaction, and the + signs are intermediate states in a forward rarefaction wave. For fixed $t$, as $x$ varies only the first and last occurence of a symbol have been printed. In this example, a forward shock $(L-M)$ overtakes a forward rarefaction $(M-R)$. The resulting interaction produces a sequence of weak backward shocks which eventually interact with each other.

With standard finite difference methods, the propagation of discontinuities is somewhat obscured by the smoothing effects of the difference equations. It is difficult to distinguish between rarefaction and shock waves. However, with the Glimm scheme a sharp discontinuity across any shock is maintained for all $t$. The probabilistic mechanism causes an uncertainty in the location and strength of the jump, but not in its existence.

\section{Proof of the Theorem}

The proof of the theorem is by induction on the approximating solutions. That is, for fixed $k$ and $h$, we state what the general pattern of the approximating solution is in the $u-v$ and $x-t$ planes at time $t=n h$, and then prove that no matter where the random points are chosen, these patterns are valid at time $t=(n+1) h$. This then enables us to study the approximate solutions at any time $t=$ const. $>0$ and to thereby obtain the necessary estimates. We assume that the reader is familiar with the results of [7], and we shall not hesitate to freely use these results without explicitly stating the exact reference.

Since our technique is rather geometrical, we will need a few lemmas on the structure of the shock curves, most of which were used in the papers [7] and [8], but were not explicitly singled out. In order to state these precisely, we shall 
need some notation. Let $P_{0}=\left(u_{0}, v_{0}\right)$ be a fixed point in the $u-v$ plane and let $v=s_{1}\left(u ; P_{0}\right)$ and $v=s_{2}\left(u ; P_{0}\right)$ denote the back and front shock curves starting at $P_{0}$, respectively. Similarly, let $v=w_{1}\left(u ; P_{0}\right)$ and $v=w_{2}\left(u ; P_{0}\right)$ denote the back and front rarefaction-wave curves through $P_{0}$. These latter two curves can be written in terms of the Riemann invariants $\mathscr{R}-\mathscr{S}$ in the alternate forms $\mathscr{S}=\mathscr{S}\left(P_{0}\right)$, $\mathscr{R}=\mathscr{R}\left(P_{0}\right)$ respectively. We can now state the following lemmas.

Lemma 1. The curve $\mathscr{S}=\mathscr{S}\left(P_{0}\right)\left(\mathscr{R}=\mathscr{R}\left(P_{0}\right)\right)$ lies entirely above the curve $v=s_{1}\left(u ; P_{0}\right)\left(v=s_{2}\left(u ; P_{0}\right)\right)$ for $u<u_{0}\left(u>u_{0}\right)$.

Lemma 2. Let $P_{1}=\left(u_{1}, v_{1}\right)$ be a point on $v=s_{1}\left(u ; P_{0}\right)\left(v=s_{2}\left(u ; P_{0}\right)\right)$; then $v=s_{1}\left(u ; P_{1}\right)\left(v=s_{2}\left(u ; P_{1}\right)\right)$ lies entirely above the curve $v=s_{1}\left(u ; P_{0}\right)\left(v=s_{2}\left(u ; P_{0}\right)\right)$ for $u<u_{1}\left(u>u_{1}\right)$.

Lemma 3. Let $C\left(P_{0}\right)\left(C^{\prime}\left(P_{0}\right)\right)$ denote the closed region between the curves $v=s_{2}\left(u ; P_{0}\right)$ and $v=w_{1}\left(u ; P_{0}\right), u \geqq u_{0}\left(v=s_{1}\left(u ; P_{0}\right)\right.$ and $\left.v=w_{2}\left(u ; P_{0}\right), u \leqq u_{0}\right)$. If $P_{1} \in C\left(P_{0}\right)\left(P_{1} \in C^{\prime}\left(P_{0}\right)\right)$, then $v=s_{2}\left(u ; P_{1}\right)\left(v=s_{1}\left(u ; P_{1}\right)\right)$ cannot meet $v=s_{2}\left(u ; P_{0}\right)$ $\left(v=s_{1}\left(u ; P_{0}\right)\right)$.

Lemma 4. Let $\Sigma\left(P_{0}\right)$ denote the closed region between the curves $v=s_{i}\left(u ; P_{0}\right)$, $i=1,2$, for $v \leqq v_{0}$. If $P_{1}=\left(u_{1}, v_{1}\right)$ is on $v=s_{1}\left(u ; P_{0}\right),\left(P_{2}\right.$ is on $\left.v=s_{2}\left(u ; P_{0}\right)\right)$, then the curve $v=s_{2}\left(u ; P_{1}\right), u \geqq u_{1}\left(v=s_{1}\left(u ; P_{2}\right), u \leqq u_{2}\right)$ cannot meet the curve $v=s_{2}\left(u ; P_{0}\right), u \geqq u_{0}\left(v=s_{1}\left(u ; P_{0}\right) u \leqq u_{0}\right)$ in $\Sigma\left(P_{0}\right)$.

The proofs of Lemmas 1 and 2 are contained in [7]; the proof of Lemma 3 is contained in [8], and the proof of Lemma 4 is similar to the proof of Lemma 3 in [5].

With these lemmas in mind we can proceed to the proof of the theorem. Let the initial data consist of the three constant values $U_{l}=\left(u_{l}, u_{l}\right), U_{m}=\left(u_{m}, v_{m}\right)$, and $U_{r}=\left(u_{r}, v_{r}\right)$ which we shall sometimes write as $L, M, R$ respectively. We let $B$ denote the largest compact region defined by the six curves $\mathscr{R}=\mathscr{R}\left(U_{l}\right), \mathscr{R}=$ $\mathscr{R}\left(U_{m}\right), \mathscr{R}=\mathscr{R}\left(U_{r}\right), \mathscr{S}=\mathscr{S}\left(U_{l}\right), \mathscr{S}=\mathscr{S}\left(U_{m}\right), \mathscr{S}=\mathscr{S}\left(U_{r}\right)$. We choose mesh lengths $k$ and $h$ so that (3) holds.

In order to prove the theorem we have to consider only two cases:

Case $A$ : forward shock wave over takes a forward rarefaction wave, and

Case $B$ : forward rarefaction wave overtakes a forward shock wave.

(The "backward" cases are similar and can be omitted.) In Case A we have two subcases: i) strong rarefaction wave $\left(\mathscr{S}\left(U_{l}\right)<\mathscr{S}\left(U_{r}\right)\right)$ and ii) weak rarefaction wave $\left(\mathscr{S}\left(U_{l}\right) \geqq \mathscr{S}\left(U_{r}\right)\right)$; see Fig. 2 .

Let us consider case i). We fix $k$ and $h$ with $k / h$ constant and assume that at time $t=n h$ the solution in the $x-t$ plane in the strip $n h<t \leqq(n+1) h$ is of the form shown in Fig. 3, that is, finitely many back shock-front rarefaction waves, followed by one back shock-front shock, followed by finitely-many front rarefaction waves. We also allow shocks and rarefaction waves of zero strength to be included in our analysis. We shall further assume that the corresponding solution in the $u-v$ plane is of the form shown by Fig. 4 . We shall show that these two diagrams are valid for the approximating solution in the strip $(n+1) h<t$ 


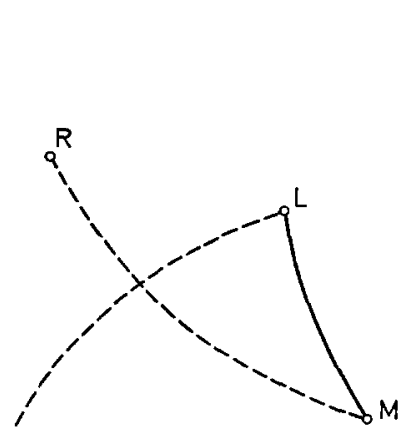

Case i): Strong rarefaction wave

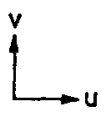

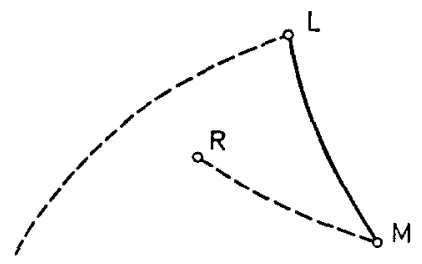

Case ii): Weak rarefaction wave.

Fig. 2. The solid lines represent the shock waves; the dotted lines represent the rarefaction waves.*

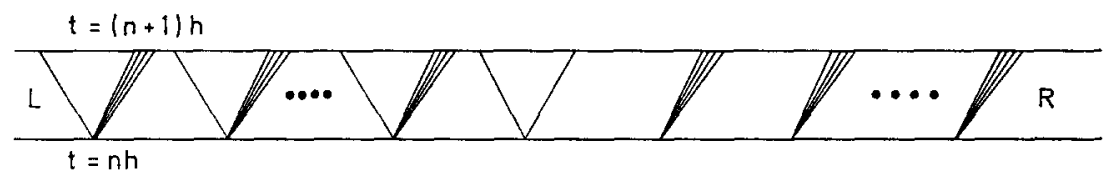

Fig. 3

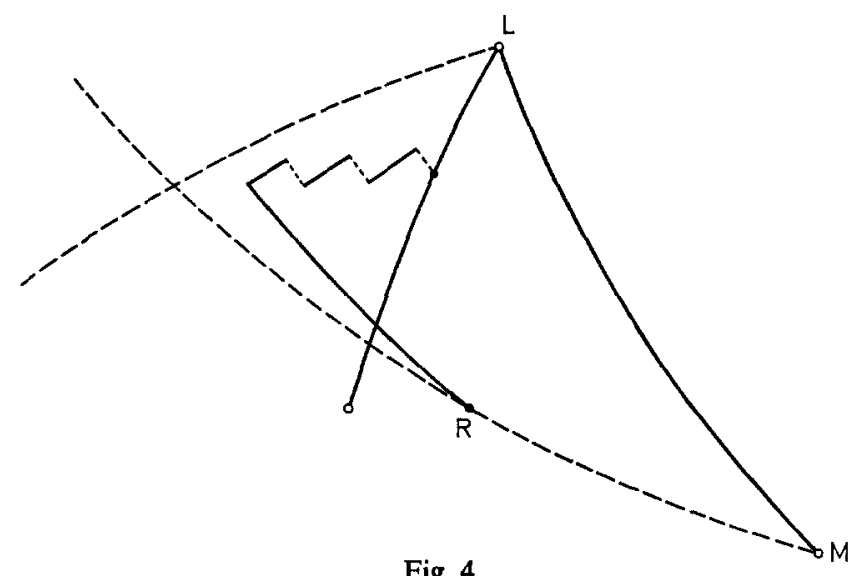

Fig. 4

$\leqq(n+2) h$, no matter where we choose the random points on the line $t=(n+1) h$. Once we do this, it will follow by induction that these diagrams are valid on every strip $n h<t \leqq(n+1) h, n=0,1,2, \ldots$.

Let $U_{m}(x)$ and $U_{m+2}(x)$ be the solutions in the adjacent rectangles [ $(m-1) k$, $(m+1) k] \times[n h,(n+1) h]$ and $[(m+1) k,(m+3) k] \times[n h,(n+1) h]$ respectively,

* The shock-wave curves are starlike with respect to the starting point, that is, each ray through the starting point meets the curve in at most one point. However, for simplicity in our diagrams, we have drawn these curves convex. 

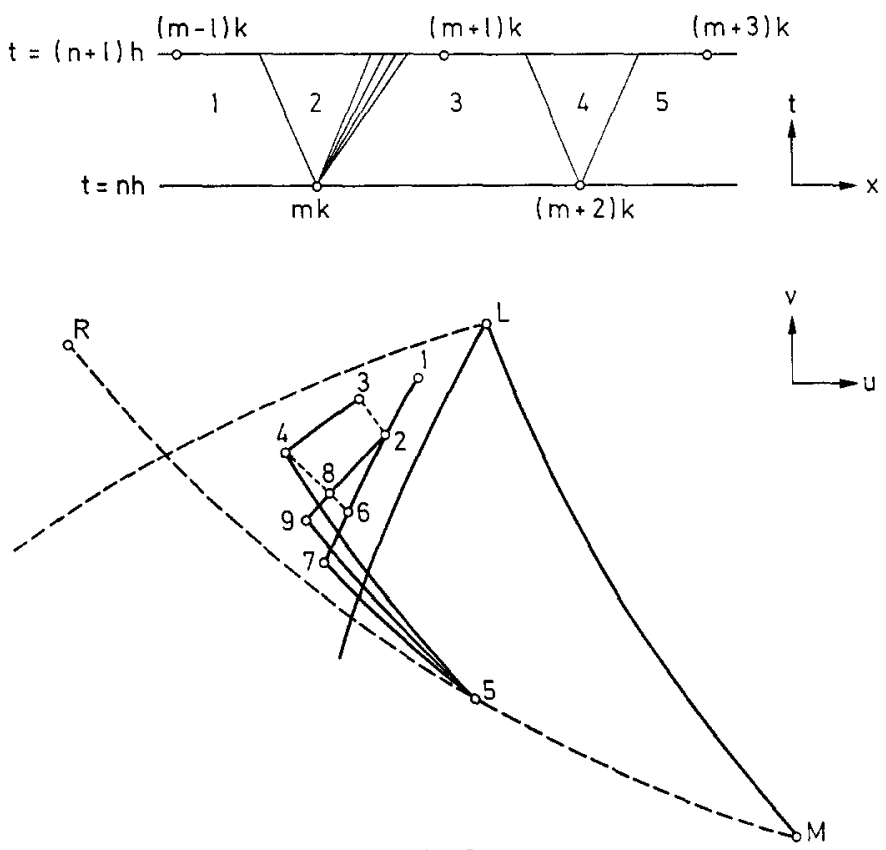

Fig. 5

and choose random points $a_{m}$ and $a_{m+2}$ on the segments $[(m-1) k,(m+1) k]$ and $[(m+1) k,(m+3) k]$ of the line $t=(n+1) h$ respectively. We are to solve the Riemann problem for (1) with initial data

$$
\left[U\left(a_{m}\right), U\left(a_{m+2}\right)\right]
$$

i.e., $U((n+1) h, x)=U\left(a_{m}\right)$, if $x<(m+1) k$, and $U((n+1) h, x)=U\left(a_{m+2}\right)$ if $x>$ $(m+1) k$. Here we have only four possibilities to consider, namely,
a) $U_{m}(x)=\left(s_{1}, w_{2}\right), U_{m+2}(x)=\left(s_{1}, w_{2}\right)$
b) $U_{m}(x)=\left(s_{1}, w_{2}\right), U_{m+2}(x)=\left(s_{1}, s_{2}\right)$
c) $U_{m}(x)=\left(s_{1}, s_{2}\right), \quad U_{m+2}(x)=w_{2}$
d) $U_{m}(x)=w_{2}, \quad U_{m+2}(x)=w_{2}$,

where we are using the obvious notations, $s_{1}=$ back shock, $w_{2}=$ front wave, etc.

Now case $d$ ) is trivial; it is resolved into waves only of the form $w_{2}$. Also from [8] it follows that the Riemann problem for (1) with data (4) in the case $a$ ) is resolved into waves of the form $\left(s_{1}, w_{2}\right)$. Next we consider case $\left.b\right)$. In order to analyze this case, we consider the diagrams in the $x-t$ and $u-v$ planes shown in Fig. 5. The random point $a_{m}$ can be chosen so that $U\left(a_{m}\right)$ is only one of the following states: (1), (2), (D) (in the rarefaction wave between (2) and (3)), or (3). Similarly $U\left(a_{m+2}\right)$ can only be (3), (4), (5). Thus we have only to consider finitely many Riemann problems which we can solve (using Lemmas 1-4). These are enumer- 

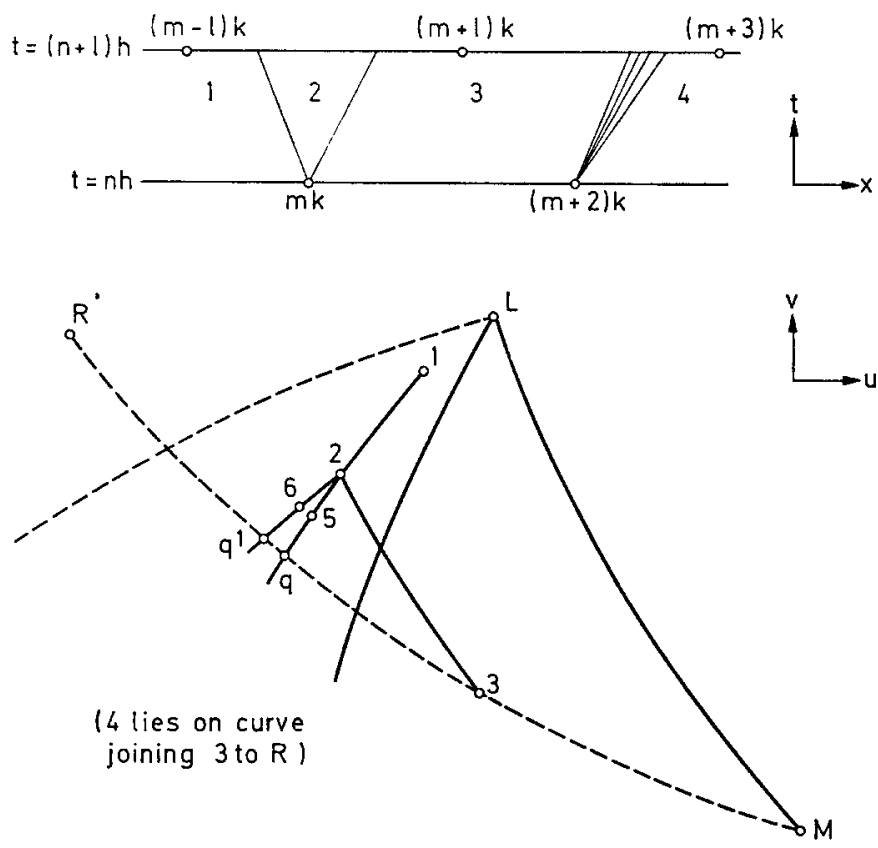

Fig. 6

ated in the table below (where the notations are self-evident, and where we omit some easy cases):

$$
\begin{aligned}
& {[(1),(3)] \rightarrow((1),(2),(3))=\left(s_{1}, w_{1}\right)} \\
& {[(1),(4)] \rightarrow((1),(6),(4))=\left(s_{1}, w_{2}\right)} \\
& {[(1),(5)] \rightarrow(\text { (1), (7), (5) })=\left(s_{1}, s_{2}\right)} \\
& {[(2),(4)] \rightarrow(\text { (2), (8), (4) })=\left(s_{1}, w_{2}\right)} \\
& {[(2),(5)] \rightarrow(\text { (2), (9), (5) })=\left(s_{1}, s_{2}\right) .}
\end{aligned}
$$

Note that if the solution is of the form $\left(s_{1}, s_{2}\right)$, then the state on the right is (5). Hence in the strip $(n+1) h<t \leqq(n+2) h$, we can only get one back shock-front shock solution.

Next we consider case $c$ ); in order to analyze this case, we consider the diagrams in the $x-t$ and $u-v$ planes shown in Fig. 6. An analysis similar to the above yields the following table (here (D) is in the rarefaction wave between (3) and (4)):

$[(1),(D)] \rightarrow((1),(5),(D))=\left(s_{1}, s_{2}\right), \quad$ if $p$ is below $q$

$[(1),(D)] \rightarrow((1),(Q),(D))=\left(s_{1}, w_{2}\right), \quad$ if $p$ is above $q$

[(2), (D)] $\rightarrow((2),(6),(D))=\left(s_{1}, s_{2}\right), \quad$ if $p$ is below $q^{\prime}$

[(2), (D) ] $\rightarrow\left((2),\left(q^{\prime}\right),(D)\right)=\left(s_{1}, w_{2}\right), \quad$ if $p$ is above $q^{\prime}$. 


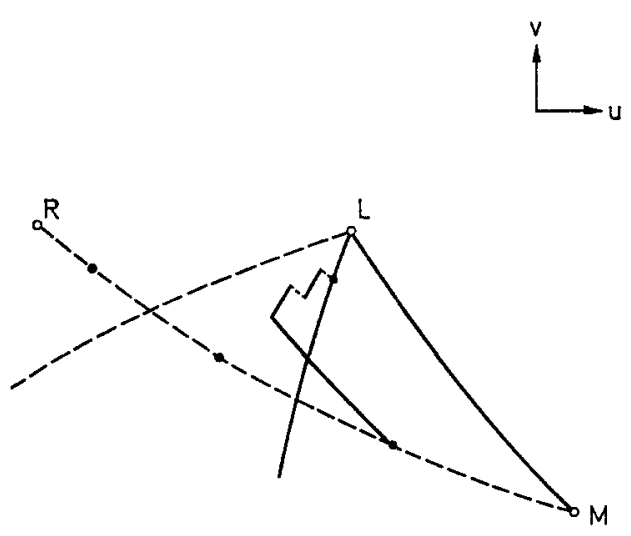

Fig. 7

Note that the solutions in cases $a$ ) through $d$ ) are of the same form in the strip $(n+1) h<t \leqq(n+2) h$ as the solution in the strip $n h<t \leqq(n+1) h$, and that the new states produced in the $u-v$ plane lie in the desired regions. Also it is easy to check that this analysis is also valid in degenerate cases; i.e. in the cases where there are front or back waves of zero strength. It follows that the induction step is proved in this case, and so Figs. 3 and 4 are valid on the line $t=(n+1) h$.

Next consider the case $i$ ) of a weak rarefaction wave (see Fig. 2). The induction proof of this case is very similar to that of case $i$ ). The solution in the $x-t$ plane in the strip $n h<t \leqq(n+1) h$ is the same as Fig. 3, and in the $u-v$ plane the solution is of the form shown in Fig. 7. We leave the verification of this to the reader.

We shall now show that a subsequence of the approximating solutions converges to a solution of the system in Case A. In order to do this, it suffices [2] to obtain an estimate of the form

$$
\operatorname{Var} U_{a, h}(t, \cdot) \leqq \text { const }
$$

where the constant is independent of the random point $a$ and the mesh length $h$. From our knowledge of the form of the solution in each strip $n h<t \leqq(n+1) h$, we shall show that this is a relatively easy task.

We first note that in both cases $i$ ) and $i i$ ) each of the approximating solutions is bounded independently of the choice of the random point $a$ and the mesh lengths $h$ and $k$. Let us now consider the variation of $u$ on any line $t=c=$ const. $>0$, for any fixed $a, h$ and $k$. We shall again only consider case $i$ ), the reason being that case ii) is similar. Suppose that the solution in the strip $\alpha h<t \leqq(\alpha+1) h$ containing $t=c$, consists of $n$ adjacent solutions $\left(U_{j}, U_{j+1}, U_{j+2}\right), j=1,2, \ldots$, $2 n-1, U_{1}=U_{l}$, of the form $\left(s_{1}, w_{2}\right)$, one $\left(s_{1}, s_{2}\right)=\left(U_{2 n+1}, U_{2 n+2}, U_{2 n+3}\right)$, and $m$ front waves $w_{2}$. Then from the diagrams of the form of Fig. 4 , we see that $u$ is decreasing along the first $n$ solutions and along the last $m$ solutions. Thus if 


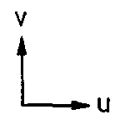

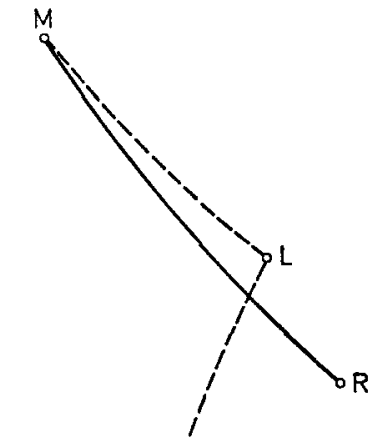

Case i): Strong shock wave

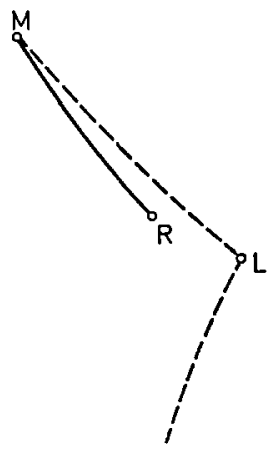

Case ii): Weak shock wave

Fig. 8

$U^{\prime}=\left(u^{\prime}, v^{\prime}\right)$ denotes the intersection of $\mathscr{S}=\mathscr{S}\left(U_{l}\right)$ with $\mathscr{R}=\mathscr{R}\left(U_{m}\right)$, we have

so that

$$
\begin{aligned}
\operatorname{Var} u_{a, h}(c, \cdot) & \leqq\left(u_{l}-u_{2 n+2}\right)+\left(u_{2 n+3}-u_{2 n+2}\right)+\left(u_{2 n+3}-u_{r}\right) \\
& \leqq\left(u_{l}-u^{\prime}\right)+2\left(u_{m}-u_{r}\right)=M_{1}
\end{aligned}
$$

$$
\operatorname{Var} u_{a, h}(c, \cdot) \leqq M_{1}
$$

Next by considering Fig. 4 we see that $\mathscr{R}$ is monotonically decreasing along $t=c$, so that

$$
\operatorname{Var} \mathscr{R}_{a, h}(c, \cdot) \leqq \mathscr{R}\left(U_{l}\right)-\mathscr{R}\left(U_{r}\right)=M_{2}
$$

Now let $\left(\mathscr{R}_{1}, \mathscr{S}_{1}\right)$ and $\left(\mathscr{R}_{2}, \mathscr{S}_{2}\right)$ be any two points in the $\mathscr{R}-\mathscr{S}$ plane and let $\Delta v=v\left(\mathscr{R}_{1}, \mathscr{S}_{1}\right)-v\left(\mathscr{R}_{2}, \mathscr{S}_{2}\right), \quad \Delta u=u\left(\mathscr{R}_{1}, \mathscr{S}_{1}\right)-u\left(\mathscr{R}_{2}, \mathscr{S}_{2}\right), \quad \Delta \mathscr{S}_{=} \mathscr{S}_{1}-\mathscr{S}_{2}, \quad \Delta \mathscr{R}=$ $\mathscr{R}_{1}-\mathscr{R}_{2}$. Then

$$
\begin{aligned}
& \Delta v=v_{\mathscr{S}}\left(\mathscr{R}_{1}, \xi\right) \Delta \mathscr{S}+v_{\mathscr{R}}\left(\eta, \mathscr{S}_{2}\right) \Delta \mathscr{R} \\
& \Delta u=u_{\mathscr{S}}\left(\mathscr{R}_{1}, \bar{\xi}\right) \Delta \mathscr{S}+u_{\mathscr{R}}\left(\bar{\eta}, \mathscr{S}_{2}\right) \Delta \mathscr{R},
\end{aligned}
$$

where $\xi$ and $\xi$ are between $\mathscr{S}_{1}$ and $\mathscr{S}_{2}, \eta$ and $\bar{\eta}$ are between $\mathscr{R}_{1}$ and $\mathscr{R}_{2}$. It follows that

$$
\begin{aligned}
(\Delta v) u_{\mathscr{S}}\left(\mathscr{R}_{1}, \bar{\xi}\right)= & (\Delta u) v_{\mathscr{S}}\left(\mathscr{R}_{1}, \xi\right) \\
& -(\Delta \mathscr{R})\left[v_{\mathscr{S}}\left(\mathscr{R}_{1}, \xi\right) u_{r}\left(\bar{\eta}, \mathscr{S}_{2}\right)-u\left(\mathscr{R}_{1}, \bar{\xi}\right) v_{\mathscr{R}}\left(\eta, \mathscr{S}_{2}\right)\right] .
\end{aligned}
$$

Hence we see from (6), (7) and the uniform boundedness of $U_{a, h}$, that if $u_{a} \neq 0$ in $B$, then we can obtain an estimate of the form

$$
\operatorname{Var} v_{a, h}(c, \cdot) \leqq M_{3}
$$




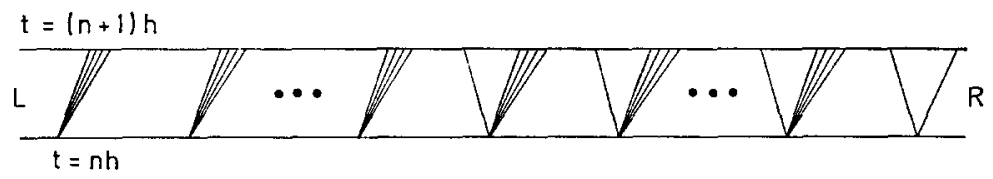

Fig. 9

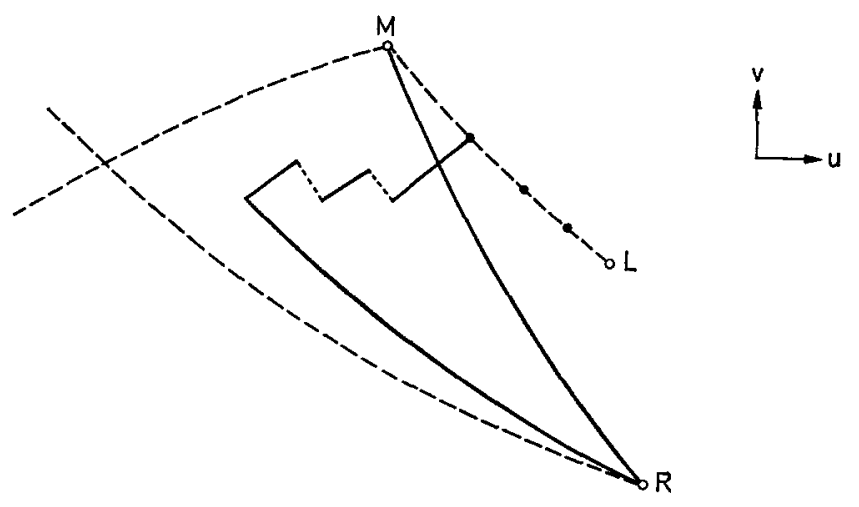

Fig. 10

and (9) together with (6) implies (5). To show that $u_{\mathscr{R}} \neq 0$, we observe that if $T$ is the mapping $T:(u, v) \rightarrow(\mathscr{R}, \mathscr{S})$, then $T$ is a $C^{1}$ bijection,

$$
d T=\left(\begin{array}{cc}
\mathscr{R}_{u} & \mathscr{R}_{v} \\
\mathscr{S}_{u} & \mathscr{S}_{v}
\end{array}\right), \quad(d T)^{-1}=\frac{1}{|d T|}\left(\begin{array}{cc}
\mathscr{S}_{v} & -\mathscr{R}_{v} \\
-\mathscr{S}_{u} & \mathscr{R}_{u}
\end{array}\right)=\left(\begin{array}{cc}
u_{\mathscr{R}} & u_{\mathscr{S}} \\
v_{\mathscr{R}} & v_{\mathscr{S}}
\end{array}\right)
$$

so that $u_{\mathscr{R}}=|d T|^{-1} \mathscr{S}_{v}$. Now the left eigenvectors of $d F$ are $\left(\mathscr{R}_{u}, \mathscr{R}_{v}\right),\left(\mathscr{S}_{u}, \mathscr{S}_{v}\right)$, and none of these are parallel to the $u$ or $v$ axes (see [7] or [8]). Therefore $\mathscr{S}_{v}$ is never zero and so $u_{\mathscr{R}}$ is never zero. This completes the proof of the theorem in Case A, a shock wave overtakes a rarefaction wave.

Let us next consider Case $B$ where a forward rarefaction wave overtakes a forward shock wave. Here again we shall distinguish two subcases: $i$ ) strong shock wave $\left(\mathscr{S}\left(U_{l}\right)>\mathscr{S}\left(U_{r}\right)\right)$ and $\left.i i\right)$ weak shock wave $\left(\mathscr{S}\left(U_{r}\right) \geqq \mathscr{S}\left(U_{l}\right)\right)$; see Fig. 8. Let us first consider case $i)$. We fix mesh lengths $h$ and $k$ and assume that at time $t=n h$ the solution in the $x-t$ plane in the strip $n h<t \leqq(n+1) h$ is of the form shown in Fig. 9, that is, finitely-many forward rarefaction waves, followed by finitely many back shock-forward rarefaction waves, followed by a back-front shock. We shall also assume that the corresponding solution in the $u-v$ plane is of the form given by Fig. 10.

It is easy to see from the considerations in Case A that in the $x-t$ plane the solution in the strip $(n+1) h<t \leqq(n+2) h$ is of the same form as in Fig. 9. Moreover, it is also easy to check that the corresponding solution in the $u-v$ plane is of the same form as in Fig. 10. Furthermore, these two statements are also valid 


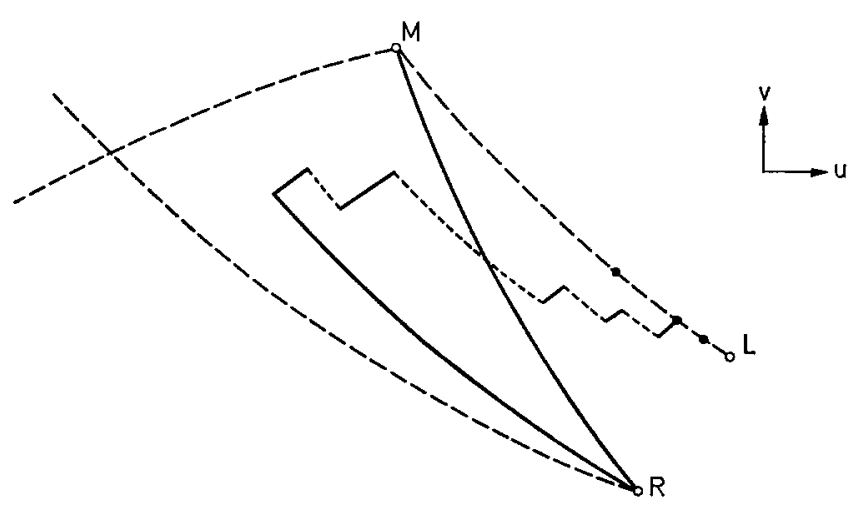

Fig. 11

in the degenerate cases of waves of zero strength. Thus one can easily verify the induction step in this case.

Let us now turn to case $i i)$. Here the results are similar. We again assume that the approximating solutions in the $x-t$ plane are of the form given in Fig. 9. The solution in the $u-v$ plane is of the form (excluding trivial cases) given by Fig. 11. Here again, one can show that these diagrams "propagate", i.e. that the solutions in the strip $(n+1) h<t \leqq(n+2) h$ are of the same form.

In order to prove that there is a convergent subsequence of approximate solutions one again obtains estimates of the form (5) by the techniques used above. We leave these details to the reader.

We conclude this paper by remarking that our methods could be extended to more general data of the form (2), although the details would be quite tedious.

C. MOLER was supported in part by the Office 'of Naval Research, Contract NR 044-377, and J. SMOLLeR by the Air Force Office of Scientific Research, Contract AFOSR-69-1662.

\section{References}

1. Courant, R., \& K. O. Friedrichs, Supersonic Flow and Shock Waves. New York: Interscience 1948.

2. Gumm, J., Solutions in the large for nonlinear hyperbolic sytems of equations. Comm. Pure Appl. Math. 18, 695-717 (1965).

3. Glimm, J., \& P. D. LAX, Decay of solutions of systems of hyperbolic conservation laws. N.Y.U. A.E.C, report, N.Y.O-1480-115, April, 1969.

4. Johnson, J. L., Global continuous solutions of hyperbolic systems of quasi-linear equations. Bull. A.M.S. 73, 639-641 (1967).

5. Johnson, J. L., \& J. A. Smoller, Global solutions of hyperbolic systems of conservation laws in two dependent variables. Bull. A.M.S. 74, 915-918 (1968).

6. NiSHIDA, T., Global solution for an initial boundary value problem of a quasilinear hyperbolic system. Proc. Jap. Acad., 44, 642-646 (1968). 
7. SMOLLER, J. A., On the solution of the Riemann problem with general step data for an extended class of hyperbolic system. Mich. Math. J. 16, 201-210 (1969).

8. SMOLler, J. A., \& J. L. Johnson, Global solutions for an extended class of hyperbolic systems of conservation laws. Arch. Rational Mech. Anal. 32, 169-189 (1969).

9. YAMAGUTI, M., \& T. NishidA, On some global solution for quasi-linear hyperbolic equations. Funk. Ekv. 11, $51-57$ (1968).

\author{
University of Michigan \\ Ann Arbor \\ Courant Institute \\ New York
}

(Received December 10, 1969) 